\title{
Adaptive Target Detection of High-range- resolution Radar in Non-Gaussian Clutter
}

\author{
Lina Pan \\ 3 Department of Basic Sciences \\ Naval Aeronautical and Astronautical University \\ Yantai, 264001, P.R. China \\ jwang67@126.com
}

Tao Jian *

1 Key Laboratory for Radar Signal Processing

Xidian University

Xi'an 710071, P.R. China

2 Research Institute of Information Fusion

Naval Aeronautical and Astronautical University

Yantai, 264001, P.R. China

work_jt@163.com

* Corresponding Author

\begin{abstract}
Adaptive target detection of high-rangeresolution radar in spherically invariant random vector clutter is investigated for a possibly singular estimated covariance matrix. Under an appropriate constraint on the unknown parameter space, the maximum likelihood estimates are obtained. By utilizing the modified recursive estimator for possibly singular covariance matrix estimation, the detector of adaptive restricted generalized likelihood ratio is devised for non-Gaussian scenarios. The results show that the proposed detector outperforms the existing Kelly binary integrator. As the number of equivalent scatterers increases, the detection performance improves whereas the performance gain decreases. Moreover, in the range of high values of detection probability, the detection performance decreases as the clutter spikiness increases, while the performance increases with increasing clutter spikes in the range of low values of detection probability.
\end{abstract}

Keywords- high-range-resolution radar; adaptive detection; non-Gaussian clutter; clutter spikiness; singularmatrix

\section{INTRODUCTION}

A high-range-resolution radar can resolve a target into a number of scatterers, by using pulse compression techniques. And the multiple dominant scatterers in isolated range cells are usually referred to as a so-called range-spread target [1]. As the detection strategies of point-like targets may fail for range-spread targets, the adaptive detection of range-spread target has gained more and more attention [2]. At present, range-spread target detection in Gaussian or partially homogeneous environment has been investigated largely [3, 4]. In fact, at the higher range resolution, the radar system receives target-like spikes representing non-Gaussian clutter observations, which can be better described as a spherically invariant random vector (SIRV) $[2,5]$.

Most of the above detectors are based on the generalized likelihood ratio (GLR) test strategy. However,

\author{
Yu Liu \\ 2 Research Institute of Information Fusion \\ Naval Aeronautical and Astronautical University \\ Yantai, 264001, P.R. China \\ hxd_hy@126.com
}

the GLRT strategy may fail to deal with this kind of problem as it is possible that a certain estimated covariance matrix can become singular [6]. In this work, the adaptive detection of range-spread target in SIRV clutter is investigated for a possibly singular estimated covariance matrix. Firstly, the restricted maximum likelihood (RML) estimates of unknown parameters are derived. And then an adaptive restricted GLR (ARGLR) detector is devised.

\section{PROBLEM FormulATION AND DETECTOR DESIGN}

Assume that data are collected from $N$ sensors and the possible target is completely contained within $K$ range cells $\mathbf{z}_{t} \mathrm{~s}, t=1, \ldots, K$. The problem of detecting a rangespread target in additive clutter can be formulated succinctly as:

$$
\begin{aligned}
& \mathrm{H}_{0}: \mathbf{Z}=\mathbf{C} \\
& \mathrm{H}_{1}: \mathbf{Z}=\mathbf{C}+\mathbf{p a}^{\mathrm{H}}
\end{aligned}
$$

where $(.)^{\mathrm{H}} \quad$ implies the conjugate transpose; $\mathbf{Z}=\left(\mathbf{z}_{1}, \mathbf{z}_{2}, \ldots, \mathbf{z}_{K}\right)$ and $\mathbf{C}=\left(\mathbf{c}_{1}, \mathbf{c}_{2}, \ldots, \mathbf{c}_{K}\right)$ denote the $N \times K$ input data matrix and clutter matrix, respectively; $\boldsymbol{\alpha}=\left(\alpha_{t}, \alpha_{2}, \ldots, \alpha_{K}\right)^{\mathrm{T}}\left((\cdot)^{\mathrm{T}}\right.$ represents the transpose $)$ and the $\alpha_{t} \mathrm{~s}, t=1, \ldots, K$ are unknown amplitudes of target scatterers; $\mathbf{p}$ denotes the normalized steering vector, such that $\mathbf{p}^{\mathrm{H}} \mathbf{p}=1$. Assume $K \geq N$.

The clutter returns are modeled as a SIRV distribution [2] for representing non-Gaussian scenarios. Thus the $N$ dimensional clutter vector $\mathbf{c}_{t}$ at range $t$ can be given by

$$
\mathbf{c}_{t}=\sqrt{\tau_{t}} \cdot \boldsymbol{\eta}_{t}, t=1, \ldots, K
$$

where $\quad \boldsymbol{\eta}_{t}=\left(\eta_{t}(1), \eta_{t}(2), \ldots, \eta_{t}(N)\right)^{\mathrm{T}} \quad$ are $\quad$ zero-mean complex Gaussian random vector with a nonnegative definite covariance matrix $\boldsymbol{\Sigma}$ with rank $r$ 
( $1 \leq r \leq N$ ).Moreover, the texture component $\tau_{t}$ is independent of $\boldsymbol{\eta}_{t}$ from range cell to range cell.

According to the previous assumptions, $\alpha_{t}, \tau_{t}$ and $\Sigma$ are unknown but $\mathbf{p}$ is known, therefore the joint conditionally probability density function of $\mathbf{Z}$ under each hypothes is is given by [6]

$$
\begin{array}{r}
f_{i}\left(\mathbf{Z} \mid \mathbf{\Sigma}, i \boldsymbol{\alpha}, \mathbf{T}, \mathrm{H}_{i}\right)=\frac{c}{\prod_{t=1}^{K}\left\|\tau_{t} \boldsymbol{\Sigma}\right\|_{\mathrm{p}}} \delta\left(\mathbf{\Phi}_{2}^{\mathrm{H}}\left(\mathbf{Z}-i \mathbf{p} \boldsymbol{\alpha}^{\mathrm{H}}\right)\right) \\
\times \exp \left(-\operatorname{tr}\left(\boldsymbol{\Sigma}^{\dagger}\left(\mathbf{Z}-i \mathbf{p} \boldsymbol{\alpha}^{\mathrm{H}}\right) \mathbf{T}^{-1}\left(\mathbf{Z}-i \mathbf{p} \boldsymbol{\alpha}^{\mathrm{H}}\right)^{\mathrm{H}}\right)\right) \\
=m_{i}(\mathbf{Z} \mid \boldsymbol{\Sigma}, \boldsymbol{\alpha}, \mathbf{T}) \delta\left(\boldsymbol{\Phi}_{2}^{\mathrm{H}}\left(\mathbf{Z}-i \mathbf{p} \boldsymbol{\alpha}^{\mathrm{H}}\right)\right)
\end{array}
$$

where $i=1,2, c$ is a constant; $\|\cdot\|_{\mathrm{p}}$ is the product of the positive eigenvalues of a square matrix; $\operatorname{tr}($.$) denotes the$ trace of a square matrix; $\boldsymbol{\Sigma}^{\dagger}$ implies the Moore-Penrose generalized inverse of $\boldsymbol{\Sigma} ; \delta($.$) is a product of Dirac delta$ functions of the matrix elements of the argument; $\boldsymbol{\Phi}_{2}$ indicates an $N \times(N-r)$ matrix whose columns form an orthonormal basis for the null space of columns of $\boldsymbol{\Sigma}$; $\mathbf{T}=\operatorname{diag}\left\{\tau_{1}, \tau_{2}, \ldots, \tau_{K}\right\}$ denotes a $K \times K$ diagonal matrix.

Due to total ignorance of $\boldsymbol{\alpha}, \mathbf{T}$ and $\boldsymbol{\Sigma}$, the GLRTbased decision schemes is used, which is tantamount to replace the unknown parameters with their maximum likelihood estimates under each hypothesis based on the entirety of data. The GLRT decision scheme is given by

$$
\frac{\sup _{\boldsymbol{\Sigma}, \mathbf{a}, \mathbf{T}} f_{1}\left(\mathbf{Z} \mid \boldsymbol{\Sigma}, \boldsymbol{\alpha}, \mathbf{T}, \mathrm{H}_{1}\right)^{\mathrm{H}_{1}}>}{\sup _{\boldsymbol{\Sigma}, \mathbf{T}} f_{0}\left(\mathbf{Z} \mid \boldsymbol{\Sigma}, \mathbf{T}, \mathrm{H}_{0}\right)} \underset{\mathrm{H}_{0}}{<} G
$$

Unfortunately, the numerator of (6) maybe unbounded, and as a consequence, the GLRT strategy cannot be applied. For example, set $\boldsymbol{\alpha}=\mathbf{Z}^{\mathrm{H}} \mathbf{S}$, and then the null space of $\mathbf{Z}-\mathbf{p \alpha}^{\mathrm{H}}$ is nonempty. This circumstance may be resulted from the case that the parameter space is too large. Nevertheless, resorting to the method of sieves [7], restrict the parameter space $(\boldsymbol{\Sigma}, \boldsymbol{\alpha}, \mathbf{T})$ to a subspace such that the ML solution exists and is unique. Under an appropriate constraint on the parameter space, the multipliers $\left(m_{0}(\mathbf{Z} \mid \mathbf{\Sigma}, \mathbf{T})\right.$ and $\left.m_{1}(\mathbf{Z} \mid \mathbf{\Sigma}, \boldsymbol{\alpha}, \mathbf{T})\right)$ of the delta functions in (5) can be maximized, and the RML estimates result.

Assume that $K \geq N$ and $\boldsymbol{\Sigma}$ is positive definite, so that $\mathbf{Z T}^{-1} \mathbf{Z}^{\mathrm{H}}$ has rank $N$ with probability 1 . It can be obtained as

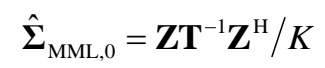

Thus

$$
\sup _{\mathbf{\Sigma}} m_{0}(\mathbf{Z} \mid \mathbf{\Sigma}, \mathbf{T})=c_{0} /\left(\left\|\mathbf{Z T}^{-1} \mathbf{Z}^{\mathrm{H}}\right\|^{K} \prod_{t=1}^{K} \tau_{t}^{N}\right)
$$

where $c_{0}$ is a constant.

To obtain the RML estimate $\hat{\boldsymbol{\Sigma}}_{\mathrm{MML}, 1}$ of $\boldsymbol{\Sigma}$ under the $\mathrm{H}_{1}$ hypothesis, restrict the parameter space to the set

$$
\mathrm{S}_{1}=\left\{\boldsymbol{\Sigma}, \boldsymbol{\alpha} \mid r_{1} \leq N-1, \boldsymbol{\Sigma}=\hat{\mathbf{\Psi}}_{1}\left[\begin{array}{cc}
\mathbf{A}_{1} & \mathbf{0}_{r_{1}, N-r_{1}} \\
\mathbf{0}_{N-r_{1}, r_{1}} & \mathbf{0}_{N-r_{1}, N-r_{1}}
\end{array}\right] \hat{\mathbf{\Psi}}_{1}^{\mathrm{H}}\right\}
$$

where $\hat{\boldsymbol{\Psi}}_{1} \boldsymbol{\Lambda}_{1} \hat{\mathbf{\Psi}}_{1}^{\mathrm{H}}$ is the eigenvalue decomposition of $\hat{\boldsymbol{\Sigma}}_{\boldsymbol{\alpha}}=\left(\mathbf{Z}-\mathbf{p} \boldsymbol{\alpha}^{\mathrm{H}}\right) \mathbf{T}^{-1}\left(\mathbf{Z}-\mathbf{p} \boldsymbol{\alpha}^{\mathrm{H}}\right)^{\mathrm{H}}$, and $\mathbf{A}_{1}$ is an $r_{1} \times r_{1}$ matrix with $r_{1}=\operatorname{rank}\left(\hat{\Sigma}_{\boldsymbol{\alpha}}\right)$.

With the constraint $S_{1}$, it can be obtained as

$$
\hat{\boldsymbol{\Sigma}}_{\mathrm{MML}, 1}=\hat{\boldsymbol{\Sigma}}_{\boldsymbol{\alpha}} / K=\left(\mathbf{Z}-\mathbf{p} \boldsymbol{\alpha}^{\mathrm{H}}\right) \mathbf{T}^{-1}\left(\mathbf{Z}-\mathbf{p} \boldsymbol{\alpha}^{\mathrm{H}}\right)^{\mathrm{H}} / K
$$

Note that $\operatorname{rank}\left(\mathbf{Z}-\mathbf{p} \boldsymbol{\alpha}^{\mathrm{H}}\right) \geq \operatorname{rank}(\mathbf{Z})-\operatorname{rank}\left(\mathbf{p} \boldsymbol{\alpha}^{\mathrm{H}}\right)=N-1$. From $(7)$, it follows that $r_{1}=\operatorname{rank}\left(\mathbf{Z}-\mathbf{p} \boldsymbol{\alpha}^{\mathrm{H}}\right)=N-1$. Hence,

$$
\sup _{\Sigma \in S_{1}} m_{1}(\mathbf{Z} \mid \Sigma, \boldsymbol{\alpha}, \mathbf{T})=c_{1} /\left(\left\|\left(\mathbf{Z}-\mathbf{p} \boldsymbol{\alpha}^{\mathrm{H}}\right) \mathbf{T}^{-1}\left(\mathbf{Z}-\mathbf{p} \boldsymbol{\alpha}^{\mathrm{H}}\right)^{\mathrm{H}}\right\|_{\mathrm{p}}^{K} \prod_{t=1}^{K} \tau_{t}^{N-1}\right)
$$

where $c_{1}$ is a constant.

The RML estimate $\hat{\boldsymbol{\alpha}}_{\mathrm{MML}}$ of $\boldsymbol{\alpha}$ under the $\mathrm{H}_{1}$ hypothesis can be written equivalently as

$$
\begin{aligned}
\hat{\boldsymbol{\alpha}}_{\mathrm{MML}} & =\arg \min _{\boldsymbol{a} \in \mathrm{S}_{1}}\left\|\left(\mathbf{Z}-\mathbf{p} \boldsymbol{\alpha}^{\mathrm{H}}\right) \mathbf{T}^{-1}\left(\mathbf{Z}-\mathbf{p} \boldsymbol{\alpha}^{\mathrm{H}}\right)^{\mathrm{H}}\right\|_{\mathrm{p}} \\
& =\arg \min _{\boldsymbol{a} \in \mathrm{S}_{1}}\left\|\left(\mathbf{X}-\mathbf{p} \mathbf{b}^{\mathrm{H}}\right)\left(\mathbf{X}-\mathbf{p b}^{\mathrm{H}}\right)^{\mathrm{H}}\right\|_{\mathrm{p}}
\end{aligned}
$$

where $\mathbf{X}=\mathbf{Z} \mathbf{T}^{-1 / 2}$ and $\mathbf{b}=\mathbf{T}^{-1 / 2} \boldsymbol{\alpha}$ with a $K \times K$ matrix $\mathbf{T}^{-1 / 2}=\operatorname{diag}\left\{1 / \sqrt{\tau_{1}}, 1 / \sqrt{\tau_{2}}, \ldots, 1 / \sqrt{\tau_{K}}\right\}$.

It follows that $\hat{\mathbf{b}}_{\mathrm{MML}}=\mathbf{X}^{\mathrm{H}} \mathbf{p}$, or equivalently $\hat{\boldsymbol{\alpha}}_{\mathrm{MML}}^{\mathrm{H}} \mathbf{T}^{-1 / 2}=\mathbf{p}^{\mathrm{H}} \mathbf{Z} \mathbf{T}^{-1 / 2}$. For the matrix $\mathbf{T}^{-1 / 2}$ is nonsingular, then $\hat{\boldsymbol{\alpha}}_{\mathrm{MML}}=\mathbf{Z}^{\mathrm{H}} \mathbf{p}$.

In fact, the ML estimate of $\tau_{t}$ is a rather difficult task and the closed-form solution does not exist [8, 9], especially in the case that a certain estimated covariance matrix could become singular. More precisely, the ML estimate of $\tau_{t}$ under each hypothesis is straightforwardly given by

$$
\begin{gathered}
\mathrm{H}_{0}: \hat{\tau}_{t, 0}=\mathbf{z}_{t}^{\mathrm{H}} \hat{\boldsymbol{\Sigma}}_{\mathrm{MML}, 0}^{\dagger} \mathbf{z}_{t} / N \\
\mathrm{H}_{1}: \hat{\tau}_{t, 1}=\left(\mathbf{z}_{t}-\hat{\alpha}_{t}^{*} \mathbf{p}\right)^{\mathrm{H}} \hat{\boldsymbol{\Sigma}}_{\mathrm{MML}, 1}^{\dagger}\left(\mathbf{z}_{t}-\hat{\alpha}_{t}^{*} \mathbf{p}\right) /(N-1)
\end{gathered}
$$

Note that, the solution of the ML estimate of $\tau_{t}$ refers to solving a transcendental equation and it cannot be obtained in closed form. Thereby, resort to a relatively simple modified recursive estimator for possibly singular covariance matrix estimation, i.e.

$$
\begin{gathered}
\hat{\tau}_{t, i}^{(k)}=\mathbf{y}_{t, i}^{\mathrm{H}}\left(\hat{\boldsymbol{\Sigma}}_{\mathrm{MML}, i}^{(k)}\right)^{\dagger} \mathbf{y}_{t, i} / r_{i} \\
\hat{\boldsymbol{\Sigma}}_{\mathrm{MML}, i}^{(k+1)}=\mathbf{Y}_{i}\left(\hat{\mathbf{T}}_{i}^{(k)}\right)^{-1} \mathbf{Y}_{i}^{\mathrm{H}} / K
\end{gathered}
$$

where $\mathbf{Y}_{i}=\left(\mathbf{y}_{1, i}, \mathbf{y}_{2, i}, \ldots, \mathbf{y}_{K, i}\right), \hat{\mathbf{T}}_{i}^{(k)}=\operatorname{diag}\left\{\hat{\tau}_{1, i}^{(k)}, \hat{\tau}_{2, i}^{(k)}, \ldots, \hat{\tau}_{K, i}^{(k)}\right\}$, for $i=0,1$ and $k=0,1, \ldots, N_{\mathrm{it}}$ with the final number of iterations $N_{\text {it }} ; \mathbf{Y}_{0}=\mathbf{Z}, r_{0}=N, \mathbf{Y}_{1}=\mathbf{Z}-\mathbf{p} \hat{\boldsymbol{\alpha}}_{\mathrm{MML}}^{\mathrm{H}}$, and $r_{1}=N-1$. Herein the recursion is initialized by the identity matrix. The resulted estimate has a satisfactory precision for $N_{\text {it }} \geq 3[8,9]$. Hereinafter, $N_{\text {it }}=3$ is adopted. Plugging the final $\hat{\mathbf{T}}_{0}^{\left(N_{\mathrm{it}}\right)}$ and $\hat{\mathbf{T}}_{1}^{\left(N_{\mathrm{it}}\right)}$ into (6) and (9), the ARGLR can be denoted as 


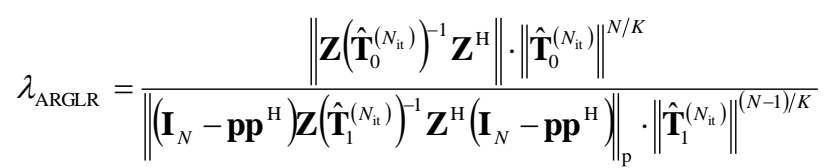

\section{PERFORMANCE ASSESSMENT}

In this section, the proposed detector is assessed and compared with the existing $M$ out of $K$ Kelly detector, which shows the effectiveness of the proposed detector.

Assume that each of all $K$ range cells has a clutter component and each of the $h_{0}$ target range cells have an equivalent signal component. Compare the proposed ARGLR with a binary integrator or $M$ out of $K$ (for short $M / K$ ) Kelly detector, which consists of two levels of detection [10]. The first level is a single-point scatterer Kelly detector [11]; the second level of detection consists of counting the number of detections from the first level detector and comparing this integer to the integer threshold $M$ of the second level detector.

The clutter samples are generated from an exponential correlation structure, i.e., Lorentzian spectrum, so that the matrix $\boldsymbol{\Sigma}$ with the one-lag correlation coefficient $\gamma$. Moreover, the texture component is modeled as a gamma distribution with the mean $b$ and the parameter $L$ controlling the deviation from Gaussian statistics. The smaller $L$ is, the more spikes will appear in clutter. Let $\sigma_{\mathrm{s}}^{2}$ and $\sigma_{c}^{2}$ indicate the average signal and clutter power per range cell respectively. The input signal-to-clutter ratio (SCR) over $K$ range cells is defined as $S C R=\sigma_{\mathrm{s}}^{2} \mathbf{p}^{\mathrm{H}} \boldsymbol{\Sigma}^{-1} \mathbf{p} / \sigma_{\mathrm{c}}^{2}$. The returns from cells of target scatterers are modeled as independent zero-mean complex circular Gaussian random variables with the variance $\varepsilon_{t} \sigma_{\mathrm{s}}^{2} K$, where $\varepsilon_{t}$ implies the fraction of the total energy returned by a given range cell $t$. Herein, five scatterers models are considered in Table I.

TABLE I. VALUES OF $\varepsilon_{t}$ FOR T YPICAL MDSMODELS

\begin{tabular}{|c|c|c|c|c|}
\hline \multirow{2}{*}{$\begin{array}{c}\text { Model } \\
\text { number }\end{array}$} & \multicolumn{4}{|c|}{ Range cell number } \\
\cline { 2 - 5 } & 1 & 2 & $\ldots$ & $h_{0}$ \\
\hline 1 & $1 / h_{0}$ & $1 / h_{0}$ & $1 / h_{0}$ & $1 / h_{0}$ \\
\hline 2 & 0.5 & $0.5 /\left(h_{0}-1\right)$ & $0.5 /\left(h_{0}-1\right)$ & $0.5 /\left(h_{0}-1\right)$ \\
\hline 3 & 0.9 & $0.1 /\left(h_{0}-1\right)$ & $0.1 /\left(h_{0}-1\right)$ & $0.1 /\left(h_{0}-1\right)$ \\
\hline 4 & 0.99 & $0.01 /\left(h_{0}-1\right)$ & $0.01 /\left(h_{0}-1\right)$ & $0.01 /\left(h_{0}-1\right)$ \\
\hline
\end{tabular}

Firstly, in Fig. 1, the effects of different scatterer distribution on the detection performance of ARGLR are evaluated, in terms of the plots of $P_{\mathrm{d}}$ versus $S C R$ for five typical models of Table 1 . It is observed that, the ARGLR performs best when the target energy is uniformly distributed, whereas the performance is degraded as the more target energy is concentrated in one cell. In fact, the more target energy is concentrated in one cell, the less range-spread target differs from a point-like target and the more collapsing loss results.

In addition, the detection performance of ARGLR with different numbers of sensors used $(N=2,4,8)$ is given in Fig. 2 , which indicates that the detection performance is improved as $N$ increases. Fig. 3 refers to different clutter preferences $(L=0.2,0.5,1,5)$, which highlights that in the range of high values of $P_{\mathrm{d}}$, the detection performance decreases as the clutter spikiness increases ( $L$ decreases), while the performance increases with increasing clutter spikes in the range of low values of $P_{\mathrm{d}}$. Note that the distribution is nearly Gaussian for $L \geq 5$.

Finally, in Fig. 4, the ARGLR is compared with the $1 / K$ Kelly detector, which indicates that the ARGLR outperforms the $1 / K$ Kelly detector. It also shows that, as the number of equivalent scatterers increases, the detection performance improves largely whereas the performance gain decreases.

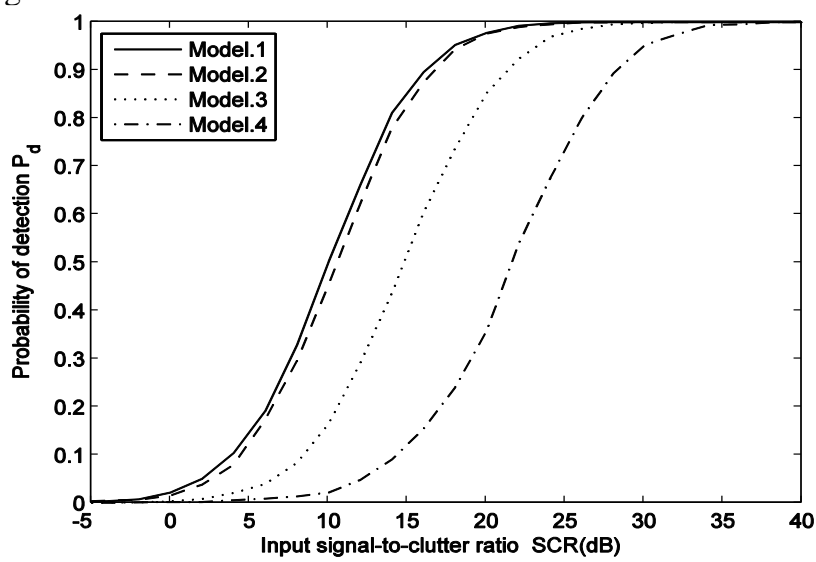

Figure 1. $P_{\mathrm{d}}$ versus $S C R$ of ARGLR for Model.1-Model.4, $P_{\mathrm{fa}}=10^{4}$, $N_{\mathrm{it}}=3, K=15, h_{0}=3, N=2, L=1, \gamma=0$

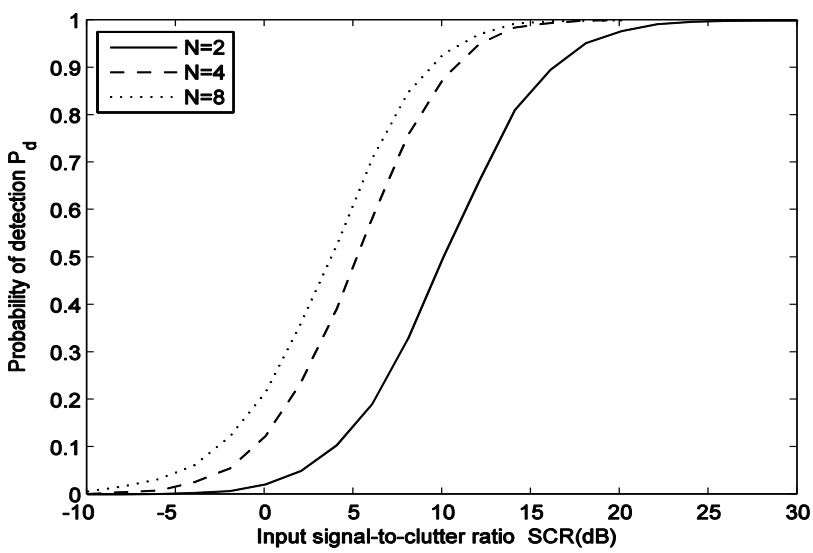

Figure 2. $P_{\mathrm{d}}$ versus $S C R$ of ARGLR for $N=2,4,8, P_{\mathrm{fa}}=10^{-4}, N_{\mathrm{it}}=3, K=15$, $h_{0}=3, L=1, \gamma=0$, Model. 1

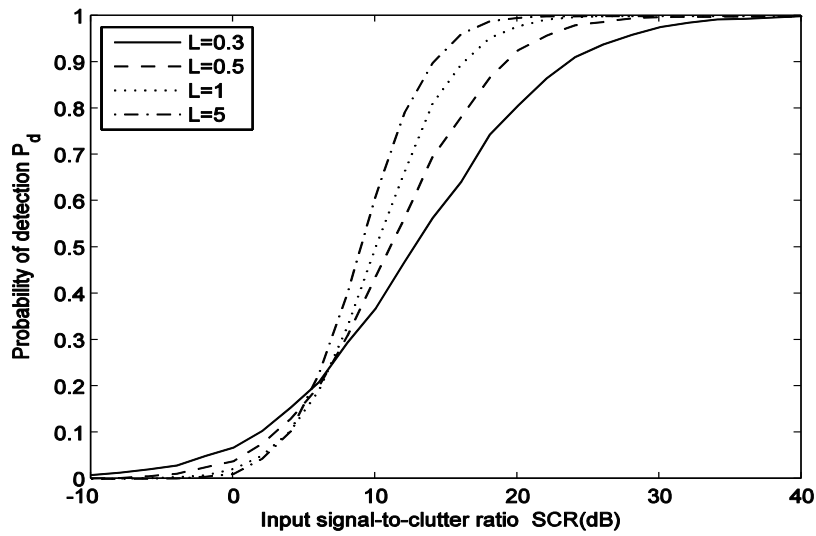

Figure 3. $P_{\mathrm{d}}$ versus $S C R$ of ARGLR for $L=0.2,0.5,1,5, P_{\mathrm{fa}}=10^{-4}, N_{\mathrm{it}}=3$, $K=15, h_{0}=3, N=2, \gamma=0$, Model. 1 


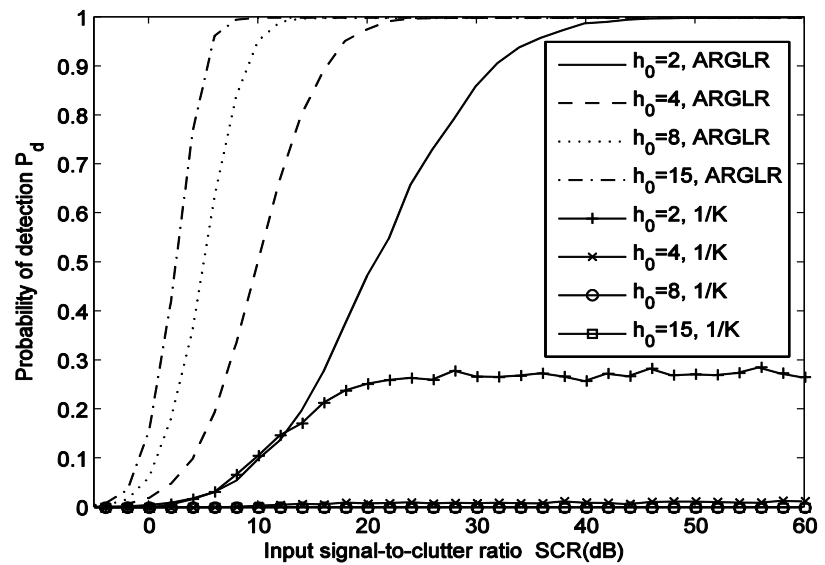

Figure 4. $P_{\mathrm{d}}$ versus $S C R$ of ARGLR and $1 / K$ Kelly detector for $h_{0}=2,4,8,15, P_{\mathrm{fa}}=10^{-4}, N_{\mathrm{it}}=3, K=15, N=2, L=1, \gamma=0$, Model. 1

\section{CONCLUSIONS}

In this paper, the adaptive detection of range-spread target in SIRV clutter is investigated for a possibly singular estimated covariance matrix. By utilizing the RML estimate and the modified recursive estimator, the ARGLR is derived for SIRV scenarios. The results show that the ARGLR outperforms the existing $M / K$ Kelly detector. As the number of equivalent scatterers increases, the detection performance improves whereas the performance gain decreases. Moreover, in the range of high values of detection probability, the detection performance decreases as the clutter spikiness increases, while the performance increases with increasing clutter spikes in the range of low values of detection probability.

\section{REFERENCES}

[1] Y. He, J. Guan and X. W. Meng, "Radar Target Detection and CFAR Processing (2nd ed.)" Beijing: Tsinghua University Press, 2011. (in Chinese)

[2] Y. He, T. Jian, F. Su, et al, "Novel range-spread target detectors in non-Gaussian clutter," IEEE Transactions on Aerospace and Electronic Systems, vol. 46, no. 3, 2010, pp. 1312-1328.

[3] F. Bandiera, A. De Maio, A. S. Greco and G. Ricci, "Adaptive radar detection of distributed targets in homogeneous and partially homogeneous noise plus subspace interference," IEEE Transactions on Signal Processing, vol. 55, no. 4, 2007, pp. 12231237.

[4] T. Jian, Y. He, F. Su, et al, "Adaptive detection of range-spread targets without secondary data in multichannel autoregressive process," Digital Signal Processing, vol. 23, no. 5, 2013, pp. 16861694.

[5] T. Jian, Y. He, F. Su, et al, "Adaptive detection of sparsely distributed target in non-Gaussian clutter," IET Radar, Sonar \& Navigation, vol. 5, no. 7,2011, pp. 780-787.

[6] K. S. Miller, "Multidimensional Gaussian Distributions," New York: Wiley, 1964.

[7] U. Grenander, “Abstract Inference,” New York: Wiley, 1981.

[8] F. Gini, and M. Greco, "Covariance matrix estimation for CFAR detection in correlated heavy tailed clutter," Signal Processing, vol. 82, no. 12,2002, pp. 1847-1859.

[9] Y. He, T. Jian, F. Su, et al, "Adaptive detection application of covariance matrix estimation for correlated non-Gaussian clutter," IEEE Transactions on Aerospace and Electronic Systems, vol. 46, no. 4, 2010, pp. 2108-2117.

[10] T. Jian, Y. He, F. Su, et al, Cascaded detector for range-spread target in non-Gaussian clutter, IEEE Transactions on Aerospace and Electronic Systems, vol. 48, no. 2, 2012,pp. 1713-1725.

[11] E. J. Kelly, "An adaptive detection algorithm," IEEE Transactions on Aerospace and Electronic Systems, vol. 22, no. 1, 1986, pp. 115-127. 